\title{
Medical radionuclide impurities in wastewater
}

\author{
T. Hay, K. Higley and D Bytwerk, \\ Dept. of Nuclear Engineering and Radiation Health Physics, Oregon State University, \\ 132 Radiation Center, Corvallis, OR 97331
}

\begin{abstract}
NCRP report No.160 states medical exposures constituted nearly half of the total radiation exposure of the US population from all sources in 2006 [1]. Part of this increase in exposure is due to the rise in nuclear medicine procedures. With this observed growth in medical radionuclide usage there is an increase in the activity being released into wastewater after medical procedures. Often medical radionuclides are not $100 \%$ radionuclide pure, but meet a certain standard of purity. Of particular interest are the longer lived impurities associated with these medical radionuclides. The longer lived impurities have a higher chance of reaching the environment. The goal of this study is to identify radionuclide impurities associated with some of the more common radiopharmaceuticals Tc-99m and I-131, locate and quantify the levels of these impurities in municipal wastewater, and make a determination as to the potential long-term impact.
\end{abstract}

\section{INTRODUCTION}

There are many studies in radioecology regarding the release of artificial radionuclides into the environment. However, there are comparably few studies addressing the release of medical radionuclides from medical facilities or following discharge of patients. Studies have shown that certain medical radionuclides are reaching the wastewater [2], and other studies have noted impurities present in radiopharmaceuticals [3]. It is important to note that typically radiopharmaceuticals are chosen for their desirable properties and relatively short half lives. However, impurities can have undesirable properties such as particulate radiation, unfavorable energy for medical imaging purposes, and longer half lives.

\subsection{Theoretical calculation of wastewater activity}

A theoretical calculation of the activity found in the wastewater was calculated using maximum allowable activity per injection $92.5 \mathrm{kBq}(\mathrm{Tc}-99 \mathrm{~m})$ and maximum allowable radionuclide impurity activity per injection (in the US) [4]. I-131 was set at an average thyroid treatment of $5 \mathrm{GBq}$ and maximum impurity noticed of $0.1 \%$ [5].

A model was created in the STELLA ${ }^{\mathrm{TM}}$ modeling software [6] to predict the activity levels of impurities in wastewater. (Note: due to space only a subset of the model is shown.) The model used current trends to extrapolate predictions for future impacts as medical radionuclide use continues to grow. Graph 1 represents the city of Corvallis (pop. 50,000) where the average number of Tc-99m procedures is around 50 per week, and the number of I-131 procedures was set at one a month representing an average month.

The model shows that it is indeed theoretically possible for the medical radionuclides and their corresponding impurities to be found in the wastewater. The graph presented above illustrates the average activity per liter and fluctuation (given as error bars) in the different treatment chambers based on the initial input of one I-131 treatment a month and 50 tc-99m treatments a week. The longer lived impurities; I-131, Te-123m and very low levels of Ru-103 are observed in the final biosolids. 


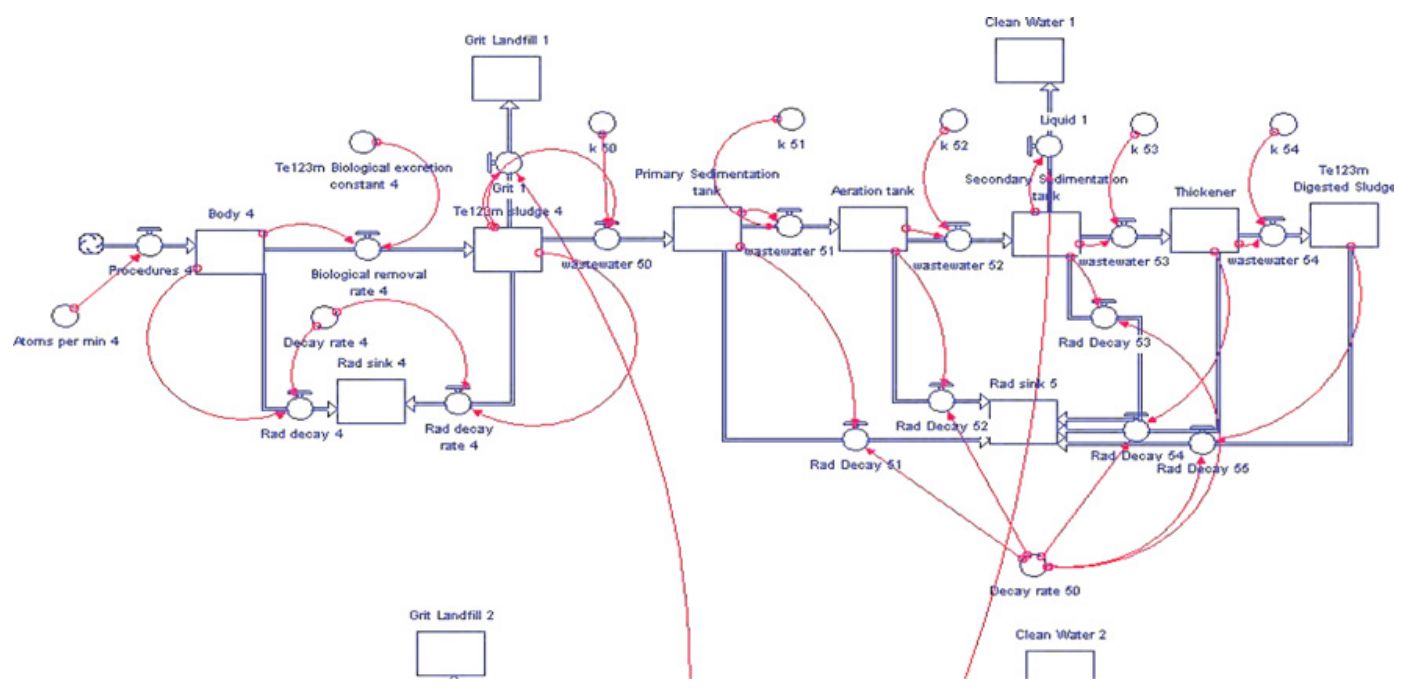

Model 1. Subset (Te-123m) of complete Stella model showing path of radionuclide from human through wastewater.

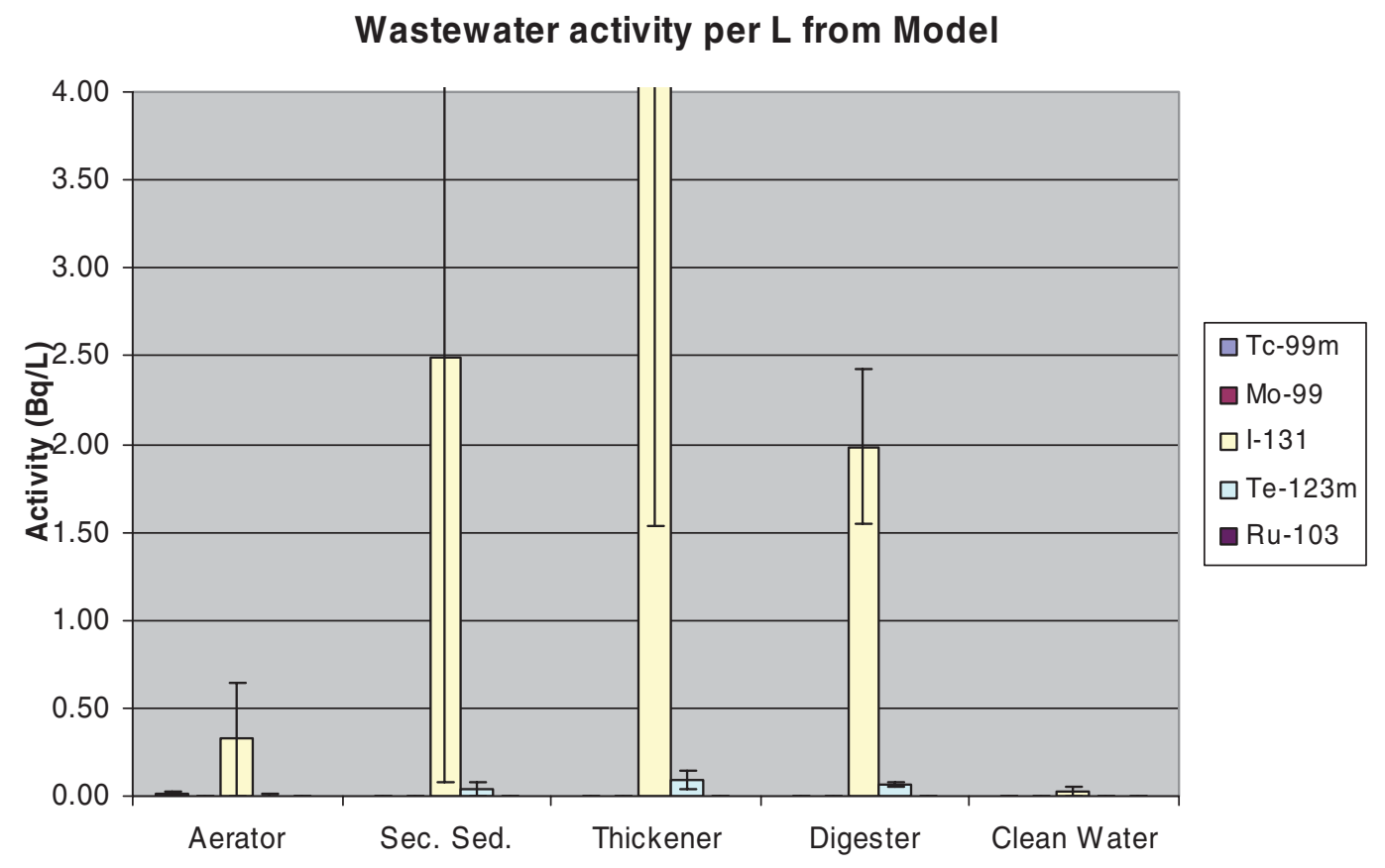

Graph 1. Model predicted activity in wastewater.

\section{MATERIALS AND METHODS}

Medical grade samples of Tc-99m and I-131 were analyzed in a High Purity Germanium Detector (HPGe) to determine if and what impurities are present. Six samples were obtained from the Corvallis 


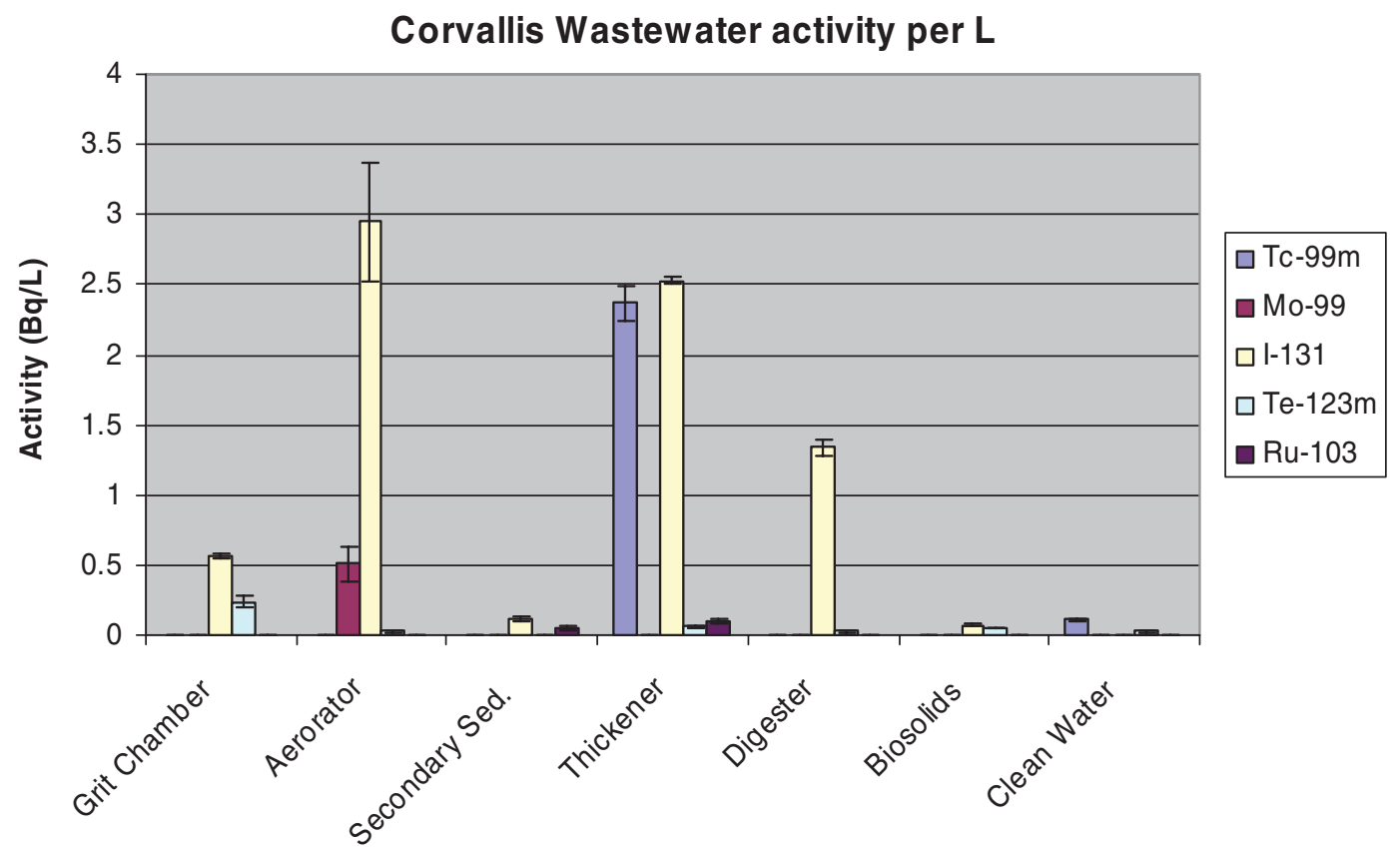

Graph 2. Analysis of the city of Corvallis wastewater given in Activity per Liter.

Wastewater Treatment Plant and seven samples were obtained from the Klamath Falls Wastewater Treatment plant. The treatment process begins at the grit chamber where the larger debris (e.g., rocks sticks, and trash) is removed and sent to the landfill. From there the wastewater is sent to the primary sedimentation where the heavier sludge is sent directly to the thickener and digester; the liquid portion heads to the activated sludge basin (aerator). Again the heavier sludge in the aerator heads to the thickener and the digester. The murky liquid is sent to the secondary treatment for its final sludge separation and finally the clean liquid is sent to the chlorine contact basin and out to the river. The sludge is sent through gravity thickeners, digested, and stored in the biosolid lagoon. In the biosolid lagoon the final sludge sits for up to six months and ultimately gets sold as fertilizer for agriculture [7]. The seven samples were taken from various points throughout the treatment process as follows:

1. Grit Chamber

2. Activated Sludge Basin

3. Secondary Treatment

4. Gravity Thickener

5. Digester

6. Biosolid Lagoon

7. Power Blowdown (Klamath only)

These samples were placed in an HPGe detector separately for three days to obtain a spectrum for each sample point. 


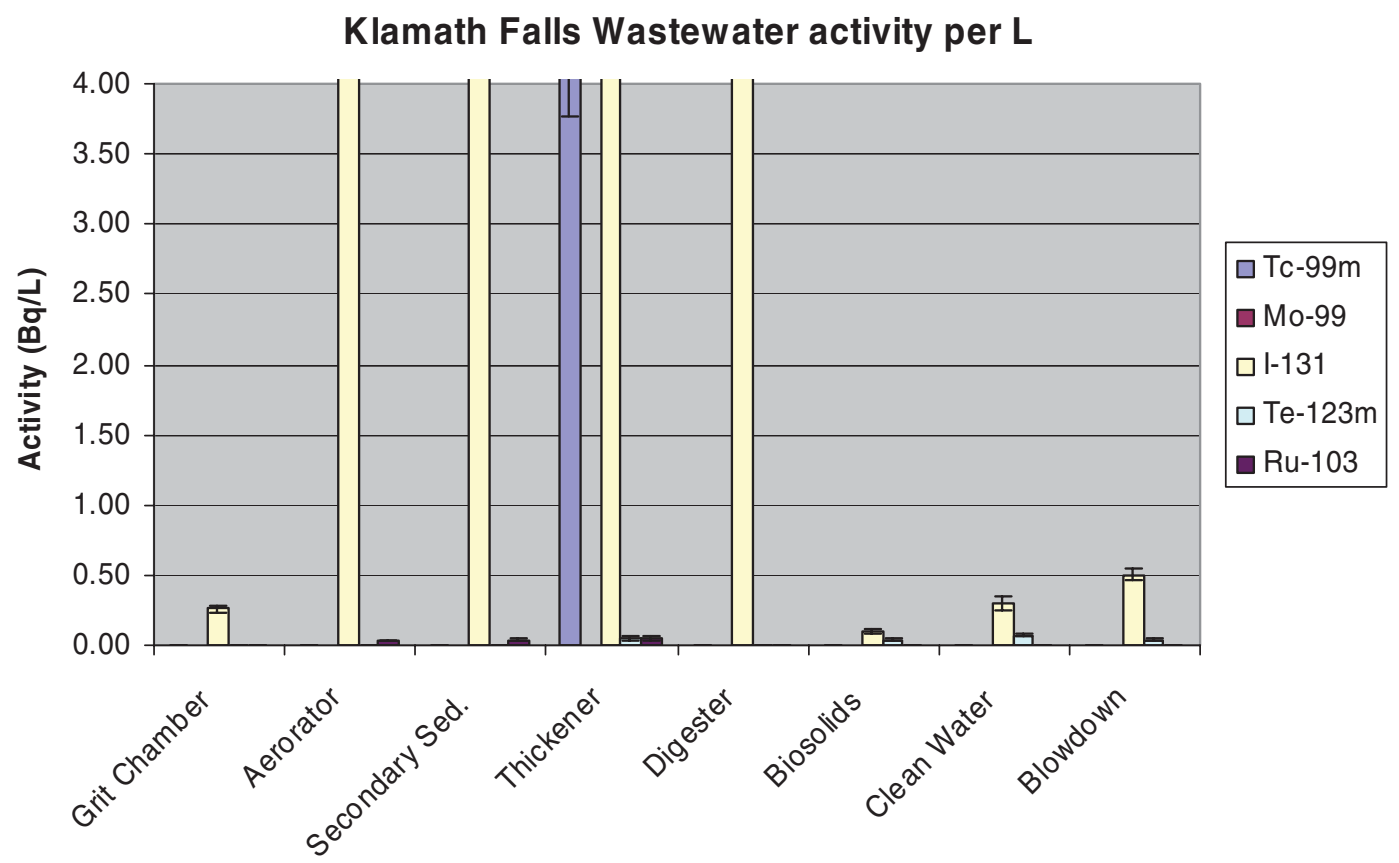

Graph 3. Analysis of the city of Klamath Falls wastewater given in Activity per Liter.

\section{RESULTS}

The spectra of the wastewater revealed that the two medical radionuclides and their associated impurities Tc-99m: Mo-99 (half life 66 h), Ru-103 (half life 40 d) and I-131: Te-123m (half life 119 d) are present at various stages during the treatment process. The analysis of the wastewater samples from both cities showed the highest concentrations of the medical radionuclides and the impurities in the thickener and the digester. Klamath Falls wastewater (Graph 3) had much more I-131 and Tc-99m present than the Corvallis wastewater (Graph 2). However, the sample taken of the final biosolids showed only trace amounts of I-131 and Te-123m. This is most likely due to the amount of time it takes the biosolids to reach the lagoon (25-30 days) and how long it sits in the lagoon (up to six months for Corvallis or held up to two months for Klamath Falls). The shorter lived nuclides are no longer detectable due to decay and dilution. It is interesting to note that I-131 was noticed in the clean water leaving the plant and in the Blowdown (recycled clean water from a local power plant), whereas in Corvallis Tc-99m was noticed in the clean water leaving the plant.

\section{CONCLUSION}

Although impurities were measured in the medical grade sample of Tc-99m and in trace amounts in the wastewater solids, the levels are so low that the likelihood of adverse effects to the environment and biota from the radiopharmaceutical impurities is minimal to none. Even with the predicted growth of nuclear medicine in the United States the levels are such that little change would be noticeable. It is also important to note that due to the time and dilution in the wastewater treatment process the impurities and the radiopharmaceutical themselves were essentially nonexistent by the time they reached the biosolid lagoon. 


\section{References}

[1] NCRP Report 160 Ionizing Radiation Exposure of the Population of the United States. (2009).

[2] Fischer, H. Ulbrich, S. Pittauerova, D. Hettwig, B. Medical Radioisotopes in the EnvironmentFollowing the Pathway from Patient to River Sediment. J. Environ. Radioact. (2009), doi:10.1016/j.jenvrad.2009.05.002

[3] DOWDALL, M. SELNÆS, O. GWYNN, J. and LIND, B. On the Use of 99Mo/99mTc Generators in the Analysis of Low Levels of 99Tc in Environmental Samples by Radiochemical Methods. Water, Air, and Soil Pollution 156 (2004) 287-297.

[4] United States Pharmecopia. The National Formulary. USP 31/NF 26th ed. (2008).

[5] Tselishchev, I. Savochkin, Yu. Mel'nik, M. Trepalina, T. Determination of radiochemical and radionuclide purity of a I-131 preparation, Journal of Radiochemistry (2007) Issue: 2

[6] Isee Systems Stella ${ }^{\mathrm{TM}}$ Modeling and Simulation Software (2011).

[7] Corvallis Public Works CPW. (2003). Corvallis Wastewater Reclamation Plant. http://www . corvallis.or.us/index . php?option=content\&task=view\&id=404\&Itemid=359 Accsessed 2010. 